\title{
Effects of Coleus Forskohlii Supplementation on Body Composition and Hematological Profiles in Mildly Overweight Women
}

\author{
Shonteh Henderson, Bahrat Magu, Chris Rasmussen, Stacey Lancaster, Chad Kerksick, Penny Smith, \\ Charlie Melton, Patty Cowan, Mike Greenwood, Conrad Earnest, Anthony Almada, Pervis Milnor, \\ Terri Magrans, Rodney Bowden, Song Ounpraseuth, Ashli Thomas, \& Richard B. Kreider
}

Exercise \& Sport Nutrition Laboratory, Baylor University, Waco, TX. Address correspondence to Richard Kreider@,baylor.edu

Received August 31, 2005/Accepted November 1, 2005

\section{ABSTRACT}

Purpose: This study investigated the effects of Coleus Forskohlii (CF) on body composition, and determined the safety and efficacy of supplementation. Methods: In a double blind and randomized manner, 23 females supplemented their diet with ForsLean ${ }^{\mathrm{TM}}(250 \mathrm{mg}$ of $10 \%$ CF extract, $(\mathrm{n}=7)$ or a placebo $[\mathrm{P}](\mathrm{n}=12)$ two times per day for 12-wks. Body composition (DEXA), body weight, and psychometric instruments were obtained at 0 , $4,8 \& 12$ weeks of supplementation. Fasting blood samples and dietary records (4-d) were obtained at 0 and 12wks. Side effects were recorded on a weekly basis. Data were analyzed by repeated measures ANOVA and are presented as mean changes from baseline for the CF and placebo groups, respectively. Results: No significant differences were observed in caloric or macronutrient intake. CF tended to mitigate gains in body mass ($0.7 \pm 1.8,1.0 \pm 2.5 \mathrm{~kg}, \mathrm{p}=0.10)$ and scanned mass $(-0.2 \pm 1.3,1.7 \pm 2.9 \mathrm{~kg}, \mathrm{p}=0.08)$ with no significant differences in fat mass $(-0.2 \pm 0.7,1.1 \pm 2.3 \mathrm{~kg}, \mathrm{p}=0.16)$, fat free mass $(-0.1 \pm 1.3,0.6 \pm 1.2 \mathrm{~kg}, \mathrm{p}=0.21)$, or body fat $(-0.2 \pm 1.0$, $0.4 \pm 1.4 \%, p=0.40)$. Subjects in the CF group tended to report less fatigue $(\mathrm{p}=0.07)$, hunger $(\mathrm{p}=0.02)$, and fullness $(\mathrm{p}=0.04)$. No clinically significant interactions were seen in metabolic markers, blood lipids, muscle and liver enzymes, electrolytes, red cells, white cells, hormones (insulin, TSH, T3, and T4), heart rate, blood pressure, or weekly reports of side effects. Conclusion: Results suggest that CF does not appear to promote weight loss but may help mitigate weight gain in overweight females with apparently no clinically significant side effects. Journal of the International Society of Sports Nutrition. 2(2):54-62, 2005

Key Words: Weight Management, Nutritional Supplement, Lipolysis, Obesity, Herbs

\section{INTRODUCTION}

Coleus forskohlii (CF) is a plant native to India. Since ancient times, plants of the Coleus species have been used as an herbal medicine to treat various disorders of the cardiovascular, respiratory, gastrointestinal, and central nervous systems (1). Forskolin has been isolated from the roots of the India-based Coleus Forskohlii. One of the most common species that contains forskolin is Coleus Forskohlii Briq. Coleus forskohlii Briq. belongs to the family Labiatae, better known as the mint family. Chemically, it is a plant rich in alkaloids, which are considered to have a high probability of influence on the biological systems (2).

Forskolin is a diterpene that acts directly on adenylate cyclase (3). Adenylate Cyclase is an enzyme that activates Cyclic Adenosine Monophosphate, or Cyclic AMP (cAMP) in the cell. Cyclic AMP promotes the breakdown of stored fats in animal and human fat cells (4). It regulates the body's thermogenic response to food, increases the body's basal metabolic rate, and increases utilization of body fat. It may also release fatty acids from adipose tissue, which results in increased thermogenesis, loss of body fat, and theoretically increased lean body mass (5). Forskolin increases cAMP accumulation, and therefore stimulates lipolysis. So, with high concentrations of forskolin, cAMP and lipolysis increase (4). Enhanced lipolysis increases fat degradation and fat usage as a fuel in the body (3). This may promote fat and weight loss. It is thought that supplementing with forskolin may enhance fat loss without loss of muscle mass (6-8). 
A previous proof-of-concept preliminary study was conducted to test the effects of CF on body composition (5). The study used a population of six overweight, but otherwise healthy, women (BMI > 25) who ingested forskolin twice daily for eight weeks. Each subject maintained her previous daily physical exercise and eating habits. The results of the study showed a significant decrease in the mean values for body weight and fat content using bioelectrical impedance (BIA) methodology. Lean body mass significantly increased compared to baseline. No side effects were reported. This preliminary study showed that, given $25 \mathrm{mg}$ of forskolin twice a day, overall body weight could improve by increasing lean body mass and by decreasing weight from body fat (5).

A recent study conducted by Godard and colleagues (6) evaluated the effects of forskolin supplementation (250 mg of a $10 \% \mathrm{CF}$ extract taken twice a day for 12-weeks) in overweight and obese men (BMI > 26) on body composition, testosterone, metabolic rate, and blood pressure. The study consisted of thirty participants randomized, in a double-blind, placebocontrolled (forskolin $n=15$, placebo $n=15$ ) manner for 12 weeks. Body composition determined by DEXA was affected due to significant decreases in fat percentage and fat mass when compared to the placebo group $(p \leq 0.05)$. Bone mass changes occurred in the forskolin group $(p \leq 0.05)$ when compared to the placebo group. The results of lean body mass revealed an increased trend when compared to the placebo group $(\mathrm{p}=0.097)$. In addition, increases in serum free testosterone occurred in the forskolin group compared to the placebo group $(\mathrm{p} \leq 0.05)$. This study showed that body composition decreased, bone mass increased, and serum free testosterone increased. Thus, it was concluded that forskolin use could possibly be used as a therapeutic agent for weight management and treatment in obese men (6).

The present study was needed to further investigate the effectiveness of CF. Aside from its potential health and therapeutic benefits, additional research was needed to assess its role in the management of body composition, and to determine the safety and efficacy of supplementation. It was also of interest to the investigator how forskolin affects general markers of health (i.e. heart rate, blood pressure, and blood variables). This study took a more comprehensive approach to explore the role that forskolin supplementation has on body composition, fat loss, and general markers of health.

\section{METHODS AND MATERIALS}

Subjects. Nineteen (19) moderately overweight (BMI of 25 - 35) female subjects between the ages of 18 and 40 participated in the study. An initial entry/familiarization session was held during which subjects completed personal and medical histories, and signed Informed Consent Statements. A general medical exam was given that included evaluating the medical history, performing a general physical examination, and evaluating whether the subjects met entry criteria to participate in the study. Entry criteria were based on age, BMI, and the presence of any medical condition deemed unsuitable for participation by the examining physician. Table 1 presents the experimental protocol/testing schedule that was conducted.

Table 1. Overview of Research Design

\begin{tabular}{|c|c|c|c|c|}
\hline Entry & $\begin{array}{l}\text { Baseline 0 } \\
\text { week (T1) }\end{array}$ & $\begin{array}{l}4 \text { week } \\
\text { (T2) }\end{array}$ & $\begin{array}{l}8 \text { week } \\
\text { (T3) }\end{array}$ & $\begin{array}{l}12 \text { week } \\
\text { (T4) }\end{array}$ \\
\hline $\begin{array}{l}\text { Phone } \\
\text { interview } \\
\text { Familiarization } \\
\text { session } \\
\text { General } \\
\text { medical exam } \\
\text { by a registered } \\
\text { nurse to } \\
\text { determine } \\
\text { qualifications } \\
\text { to participate } \\
\text { in study. }\end{array}$ & $\begin{array}{l}\text { Blood } \\
\text { Collection } \\
\text { * Clinical } \\
\quad \text { Chemistry } \\
\text { * Thyroid } \\
\quad \text { Panel } \\
\text { * Fasting } \\
\quad \text { Insulin } \\
\text { Total Body } \\
\text { Water } \\
\text { DEXA Body } \\
\text { Comp } \\
\text { HR/Blood } \\
\text { Pressure } \\
\text { Dietary } \\
\text { History } \\
\text { Questionnaires }\end{array}$ & $\begin{array}{l}\text { Total Body } \\
\text { Water } \\
\text { DEXA Body } \\
\text { Comp } \\
\text { HR/Blood } \\
\text { Pressure } \\
\text { Questionnaires }\end{array}$ & $\begin{array}{l}\text { Total Body } \\
\text { Water } \\
\text { DEXA Body } \\
\text { Comp } \\
\text { HR/Blood } \\
\text { Pressure } \\
\text { Questionnaires }\end{array}$ & $\begin{array}{l}\text { General } \\
\text { Medical Exam } \\
\text { Blood } \\
\text { Collection } \\
\text { * Clinical } \\
\text { Chemistry } \\
\text { *Thyroid } \\
\text { Panel } \\
\text { * Fasting } \\
\text { Insulin } \\
\text { Total Body } \\
\text { Water } \\
\text { DEXA Body } \\
\text { Comp } \\
\text { HR/Blood } \\
\text { Pressure } \\
\text { Dietary } \\
\text { History } \\
\text { Questionnaires }\end{array}$ \\
\hline
\end{tabular}

Pre-Supplementation / Baseline Testing. Subjects recorded all food intake on dietary record forms for four days (4-d) prior to pre-supplementation testing. Subjects were instructed to refrain from exercise for 48 hours and to fast for 8 hours prior to presupplementation testing. Subjects then reported for the initial battery of assessments, which included an appetite questionnaire, a psychological mood inventory, and the following measurements: body weight, total body water, body composition, resting heart rate, and blood pressure. Subjects then donated approximately 30 milliliters (6 teaspoons) of blood.

Supplementation Protocol. After baseline testing, subjects were randomly and blindly assigned to ingest Forslean ${ }^{\mathrm{TM}}$ or a matched placebo for 12 weeks. The Forslean ${ }^{\mathrm{TM}}$ forskolin extract and appropriate placebo were prepared by Sabinsa Corporation (Piscataway, NJ). Capsules were provided in a 2piece hard shell capsule form, and delivered in blindly labeled bottles containing 60 capsules each. 
Each capsule contained $250 \mathrm{mg}$ of the extract standardized for $10 \%$ forskolin, or a suitable placebo. Participants were instructed to take one capsule in the morning and one in the evening, a half an hour before a meal for 12 weeks.

Post-Supplementation Assessments. Subjects were instructed to provide weekly reports to the research nurse in order to monitor safety and side effects. Subjects who did not provide a weekly report were called by the research nurse to determine whether they had experienced any unexpected problems and/or adverse events. Following 4 and 8 weeks of supplementation, subjects returned to have the same initial battery of assessments performed minus the blood draw and dietary history. After 12 weeks of supplementation, subjects returned to repeat all baseline tests (including dietary history and blood draw).

\section{PROCEDURES}

Medical Monitoring. Subjects who met eligibility criteria were informed to report any unexpected problems or adverse events they encountered during the course of the study. The subjects were contacted on a weekly basis to determine if they experienced any unexpected problems and/or adverse events. If clinically significant side effects were reported, the subjects were to discuss the problem with the supervising physician to determine whether any medical treatment was needed and/or whether the subject could continue in the study. If so, the subject would be referred to obtain medical treatment from his/her personal physician. New findings and/or medical referrals of unexpected problems and/or adverse events would be documented, placed in the subjects research file, and reported to the University of Memphis IRB committee.

Dietary Inventories. Subjects recorded all food and fluid intake on dietary record forms. Dietary intake was assessed using the Food Processor III Nutrition Software (Version 7.5) (Salem, OR).

Appetite Scale. Appetite was assessed using a visual analogue scale ranging from low to high (9). This scale was used to record appetite, hunger, fullness, satisfaction, energy, and quality of food.

Psychometric Inventory. Mood changes were assessed by the Profile of Moods States (POMS) inventory (San Diego, CA). The POMS is a validated, standardized self-rating scale consisting of 57 items that measures six identifiable mood states; Tension-Anxiety; Depression-Dejection; Anger-
Hostility; Vigor-Activity; Fatigue-Inertia; ConfusionBewilderment. A 5-point scale is used from $0=$ not at all to $4=$ extremely (10).

Total Body Weight. Prior to each assessment, height was measured with the shoes removed using standard anthropometrics. Total body weight was also measured with the shoes removed using a calibrated electronic scale with a precision of $+/-0.02 \mathrm{~kg}$ (Healthometer, Bridgeview, IL). The scale was calibrated by placing certified $25-\mathrm{kg}$ weights and balancing the scale.

Total Body Water. Total body water was estimated using the Valhalla Scientific Bioelectrical Impedance Analyzer (Model 1990B, San Diego, CA), which measures bio-resistance of water and body tissues based on a minute low-energy, high frequency transmitted through the body. The analyzer was calibrated internally to a standard electrical current by pressing the calibration key located on the unit. After calibration was completed, subjects were placed in a supine position with the arms slightly bent at the elbows and palms facing down. The arms and hands were not to contact the body, and the legs were not allowed to touch each other. The subjects were instructed to remove the right shoe and sock. The laboratory technician sterilized the area where the electrodes were to be placed with a Kendall Webcol ${ }^{\mathrm{TM}}$ alcohol prep saturated with $70 \%$ isopropyl alcohol. Electrodes were placed on the posterior aspect of the hand and wrist, and on the anterior aspect of the foot and ankle.

Body Composition \& Bone Density. Body composition was determined using a calibrated Hologic 4500W dual-energy x-ray absorptiometry (Bedford, MA) with the Hologic version V7, Rev F software (Waltham, MA). The dual-energy x-ray absorptiometry (DEXA) segments regions of the body (right arm, left arm, trunk, right leg, and left leg) into three compartments for determination of fat, soft tissue (muscle), and bone mass. The scanned bone, fat, and fat-free/soft tissue mass for each region were subtotaled to determine whole body values. Percent body fat was calculated by dividing the amount of measured fat mass by total scanned mass. Day-to-day reliability studies of hip, spine, and whole body scans on men and women show the DEXA used in this study to be a highly reliable and precise method for determining variations in body composition segments (11). Test-retest reliability studies performed on male and athletes with this DEXA machine yielded a mean deviation for total BMC and total fat free/soft tissue mass of $0.31 \%$ with a mean intra-class correlation of 0.985 (11). Quality 
control (QC) calibration procedures were performed on a spine phantom (Hologic X-CALIBER Model $D P A / Q P R-1$ anthropometric spine phantom) prior to each testing session. In addition, weekly calibration procedures were performed on a density step calibration phantom. Testing was performed by certified radiology technicians who properly positioned the subjects in a supine manner on the DEXA table and executed testing according to standard procedures.

Heart Rate \& Blood Pressure. Heart rate was determined by palpitation of the radial artery according to procedures outlined in the ACSM's Guidelines for Exercise Testing and Prescription (12). Blood pressure was assessed in the supine position after resting for 5-min with a mercurial sphygmomanometer (Trimline by PyMah Corporation, Somerville, NJ) using standard procedures.

Blood Samples. Subjects observed an overnight eight (8) hour fast prior to reporting to the lab to donate blood. Approximately 6 teaspoons of venous blood (30 milliliters) were obtained through venipuncture of an antecubital vein in the forearm using standard phlebotomy procedures. Samples were collected into one (1) $10 \mathrm{~mL}$ Vacutainer $\mathrm{SST}^{\mathrm{TM}}$ gel and clot activator tube, and two (2) $5 \mathrm{~mL}$ Vacutainer Brand $\mathrm{K}_{3}$ EDTA $^{\mathrm{TM}}$ sterile interior tubes. Trained laboratory technicians centrifuged the blood samples at $5000 \mathrm{rev}$ $\mathrm{x} \min ^{-1}$ for 10 minutes in a Biofuge 17R Centrifuge (Heraeus Inc., Germany). Serum from one SST tube was transferred into Costar microcentrifuge tubes (Corning Incorporated, Corning, NY) using plastic disposable Falcon ${ }^{\mathrm{TM}}$ transfer pipets (Becton Dickinson) and frozen at $-80^{\circ}$ Celsius for subsequent analysis. Serum from the remaining SST and EDTA tubes were transferred into two (2) separate $10 \mathrm{~mL}$ plain sterile tubes. The whole blood was diluted with $2 \mathrm{~mL}$ of saline solution. Both serum and whole blood samples were refrigerated and sent to Quest Diagnostic Labs (Ann Arbor, MI) for clinical analysis.

A complete 31-panel clinical chemistry profile was run on serum samples using the Technicon DAX model 96-0147 automated chemistry analyzer (Technicon Inc., Terry Town, NY) following standard clinical procedures. This panel consisted of muscle enzymes [creatine kinase, phosphorus, calcium]; liver enzymes [direct bilirubin, total bilirubin, gamma glutamyl transferase (GGT), lactate dehydrogenase (LDH), alkaline phosphatase, AST (SGOT), ALT (SGPT)]; lipid profile [triglycerides, total cholesterol, high-density lipoprotein-cholesterol (HDL-C), lowdensity lipoprotein-cholesterol (LDL-C), cholesterol/HDLC ratio, glucose], electrolytes [sodium, potassium, chloride, carbon dioxide, urea nitrogen (BUN), creatinine, BUN/creatinine ratio], protein status [uric acid, total protein, albumin, globulin, albumin/globulin ratio], and whole blood cell counts [hemoglobin, hematocrit, red blood cell counts, white blood cell counts (basophils, absolute basophils, eosinophils, absolute eosinophils, monocytes, absolute monocytes, lymphocytes, absolute lymphocytes, neutrophils, absolute neutrophils), platelet count, mean corpuscular volume $(\mathrm{MCV})$, mean corpuscular hemoglobin $(\mathrm{MCH})$, mean corpuscular hemoglobin concentration (MCHC), red cell distribution width (RDW)]. Cell counts with percent differentials were run on whole blood samples using a Coulter STKS automated analyzer (Coulter Inc., Hialeah, FL) using standard procedures.

Frozen serum samples were sent to the Department of Physiology at East Tennessee State University to assay thyroid stimulating hormone (thyrotropin), thyroxin, total thyroxin, and fasting insulin. A chemistry profile was run on these samples using an Immulite Mark 5 HSS chemiluminescence random access immunoassay analyzer (Diagnostic Products Corporation, Los Angeles, CA) following standard procedures. These analyzers were calibrated daily to controls according to manufacturer recommendations and federal guidelines for clinical diagnostic laboratories. Test to test reliability of performing these assays ranged from 2 to $6 \%$ for individual assays with a average variation of $\pm 3 \%$. Samples were run in duplicate to verify results if the observed values were outside control values and/or clinical norms according to standard procedures (13). Analysis of these blood parameters helped determine the safety effects of this nutritional supplementation formulation on general markers of clinical health status and selected hormones.

\section{DATA ANALYSIS}

A 2 (groups) $\mathrm{x} 4$ (times) analysis of variance (ANOVA) test with repeated measures on the second factor was performed on five variables: total body weight, total body water, body composition, appetite surveys, and psychological mood state inventories. A $2 \times 2$ ANOVA test with repeated measures on the second factor was performed on the diet logs and the clinical profiles for the blood samples. Type I error was controlled at 0.05 for all of the data analysis 
Table 2. Four-day total dietary intake for the $\mathrm{CF}$ and $\mathrm{P}$ groups.

\begin{tabular}{|c|c|c|c|c|c|c|}
\hline Variable & Group & $\begin{array}{c}\text { Week 0 } \\
\text { (T1) }\end{array}$ & $\begin{array}{c}\text { Week } 8 \\
\text { (T3) } \\
\end{array}$ & $\begin{array}{c}\text { Week 12 } \\
\text { (T4) }\end{array}$ & Significance & \\
\hline $\begin{array}{l}\text { Fat } \\
(\mathrm{g} / \mathrm{kg} / \mathrm{d})\end{array}$ & $\begin{array}{c}\mathrm{CF} \\
\mathrm{P}\end{array}$ & $\begin{array}{c}281.5 \pm 87.5 \\
337.0 \pm 124.2\end{array}$ & $\begin{array}{l}243.2 \pm 69.0 \\
310.1 \pm 99.1\end{array}$ & $\begin{array}{l}187.0 \pm 55.8 \\
219.0 \pm 86.3\end{array}$ & $\begin{array}{r}\text { Group } \\
\text { Time } \\
\text { Group x Time }\end{array}$ & $\begin{array}{l}0.153 \\
0.002 \\
0.696\end{array}$ \\
\hline $\begin{array}{l}\text { Carbohydrates } \\
(\mathrm{g} / \mathrm{kg} / \mathrm{d})\end{array}$ & $\begin{array}{c}\mathrm{CF} \\
\mathrm{P}\end{array}$ & $\begin{array}{l}959.1 \pm 278.7 \\
959.7 \pm 253.6\end{array}$ & $\begin{array}{l}903.5 \pm 227.9 \\
953.3 \pm 269.2\end{array}$ & $\begin{array}{l}777.5 \pm 303.2 \\
831.5 \pm 184.1\end{array}$ & $\begin{array}{r}\text { Group } \\
\text { Time } \\
\text { Group } x \text { Time }\end{array}$ & $\begin{array}{l}0.719 \\
0.025 \\
0.676\end{array}$ \\
\hline $\begin{array}{l}\text { Protein } \\
(\mathrm{g} / \mathrm{kg} / \mathrm{d})\end{array}$ & $\begin{array}{c}\mathrm{CF} \\
\mathrm{P}\end{array}$ & $\begin{array}{l}293.8 \pm 112.0 \\
318.5 \pm 143.5\end{array}$ & $\begin{array}{c}270.8 \pm 93.0 \\
290.7 \pm 129.9\end{array}$ & $\begin{array}{l}242.8 \pm 80.7 \\
282.1 \pm 77.6\end{array}$ & $\begin{array}{r}\text { Group } \\
\text { Time } \\
\text { Group x Time }\end{array}$ & $\begin{array}{l}0.525 \\
0.213 \\
0.831\end{array}$ \\
\hline $\begin{array}{l}\text { Energy Intake } \\
(\mathrm{kcal} / \mathrm{kg} / \mathrm{d})\end{array}$ & $\begin{array}{l}\mathrm{CF} \\
\mathrm{P}\end{array}$ & $\begin{array}{l}7458 \pm 1920 \\
8087 \pm 2310\end{array}$ & $\begin{array}{l}6833 \pm 1259 \\
7772 \pm 2165\end{array}$ & $\begin{array}{l}5690 \pm 1927 \\
6435 \pm 1475\end{array}$ & $\begin{array}{r}\text { Group } \\
\text { Time } \\
\text { Group } x \text { Time }\end{array}$ & $\begin{array}{l}0.299 \\
0.007 \\
0.919\end{array}$ \\
\hline
\end{tabular}

Table 3. Selected hematological markers for the $\mathrm{CF}$ and $\mathrm{P}$ groups.

\begin{tabular}{|c|c|c|c|c|c|}
\hline Variable & Group & $\begin{array}{c}\text { Week 0 } \\
\text { (T1) }\end{array}$ & $\begin{array}{c}\text { Week 12 } \\
\text { (T4) }\end{array}$ & \multicolumn{2}{|c|}{ Significance } \\
\hline $\begin{array}{l}\text { White Blood } \\
\text { Cells } \\
\text { (thous/cum) }\end{array}$ & $\begin{array}{l}\mathrm{CF} \\
\mathrm{P}\end{array}$ & $\begin{array}{l}6.5 \pm 1.8 \\
5.9 \pm 1.7\end{array}$ & $\begin{array}{l}7.9 \pm 2.3 \\
5.1 \pm 1.7\end{array}$ & $\begin{array}{l}\text { Group } \\
\text { Time } \\
\text { Group x Time }\end{array}$ & $\begin{array}{l}0.030 \\
0.366 \\
0.007\end{array}$ \\
\hline $\begin{array}{l}\text { Absolute } \\
\text { Lymphocytes } \\
\text { (cells/mcl) }\end{array}$ & $\begin{array}{l}\mathrm{CF} \\
\mathrm{P}\end{array}$ & $\begin{array}{l}2161.2 \pm 433.6 \\
2084.4 \pm 511.4\end{array}$ & $\begin{array}{l}2771.0 \pm 643.2 \\
1945.8 \pm 447.5\end{array}$ & $\begin{array}{l}\text { Group } \\
\text { Time } \\
\text { Group x Time }\end{array}$ & $\begin{array}{l}0.051 \\
0.043 \\
0.003\end{array}$ \\
\hline $\begin{array}{l}\text { Calcium } \\
(\mathrm{mg} / \mathrm{dl})\end{array}$ & $\begin{array}{l}\mathrm{CF} \\
\mathrm{P}\end{array}$ & $\begin{array}{l}9.1 \pm 0.1 \\
9.3 \pm 0.2\end{array}$ & $\begin{array}{l}9.3 \pm 0.4 \\
9.1 \pm 0.2\end{array}$ & $\begin{array}{l}\text { Group } \\
\text { Time } \\
\text { Group x Time }\end{array}$ & $\begin{array}{l}0.894 \\
0.904 \\
0.030\end{array}$ \\
\hline $\begin{array}{l}\text { ALT (SGPT) } \\
(\mathrm{U} / \mathrm{L})\end{array}$ & $\begin{array}{l}\mathrm{CF} \\
\mathrm{P}\end{array}$ & $\begin{array}{l}11.8 \pm 4.6 \\
22.5 \pm 10.1\end{array}$ & $\begin{array}{l}11.4 \pm 3.4 \\
22.0 \pm 13.8\end{array}$ & $\begin{array}{l}\text { Group } \\
\text { Time } \\
\text { Group x Time }\end{array}$ & $\begin{array}{l}0.020 \\
0.036 \\
0.000\end{array}$ \\
\hline $\begin{array}{l}\text { Uric Acid } \\
(\mathrm{mg} / \mathrm{dl})\end{array}$ & $\begin{array}{l}\mathrm{CF} \\
\mathrm{P}\end{array}$ & $\begin{array}{l}4.2 \pm 0.9 \\
3.9 \pm 1.1\end{array}$ & $\begin{array}{l}3.8 \pm 1.0 \\
4.4 \pm 1.4\end{array}$ & $\begin{array}{l}\text { Group } \\
\text { Time } \\
\text { Group x Time }\end{array}$ & $\begin{array}{l}0.006 \\
0.724 \\
0.003\end{array}$ \\
\hline $\begin{array}{l}\text { Absolute } \\
\text { Neutrophils } \\
\text { (cells/mcl) }\end{array}$ & $\begin{array}{l}\mathrm{CF} \\
\mathrm{P}\end{array}$ & $\begin{array}{l}3791.9 \pm 1273.0 \\
3270.7 \pm 1251.3\end{array}$ & $\begin{array}{l}4467.7 \pm 1737.2 \\
2732.5 \pm 596.0\end{array}$ & $\begin{array}{l}\text { Group } \\
\text { Time } \\
\text { Group x Time }\end{array}$ & $\begin{array}{l}0.042 \\
0.787 \\
0.027\end{array}$ \\
\hline
\end{tabular}

procedures. Tukey least significant difference (LSD) post-hoc procedures were conducted when a significance level was observed. Delta values were calculated on body composition variables to further highlight significant changes that occurred during the study.

\section{RESULTS}

Nutritional Intake. Table 2 presents four day total nutritional intake data for the $\mathrm{CF}$ and $\mathrm{P}$ groups. No significant group interactions $(p>0.05)$ were observed in mean relative daily energy intake, carbohydrate intake, protein intake, or fat intake. However, daily intake for the energy, carbohydrate, and fat variables significantly decreased $(p<0.05)$ from Week 0 to Week 12.

Medical Safety Analysis. Table 3 presents significant blood markers obtained throughout the study. These hematological responses were measured for the analysis of the safety of the supplement on general markers of health. The results of the analysis indicated a significant group interaction in white blood cell levels $(p=0.007)$, absolute lymphocyte levels $(p=0.003)$, absolute neutrophils $(p=0.027)$, calcium levels $(p=0.03)$, ALT $(p=0.000)$, and uric acid $(p=0.003)$ for the CF group. Additionally, a significant group effect $(p<0.05)$ was observed in red blood cell count, \% hematocrit, $\mathrm{MCV}, \mathrm{MCH}$, 
Table 4. Body composition and bone density values for the $\mathrm{CF}$ and $\mathrm{P}$ groups

\begin{tabular}{|c|c|c|c|c|c|c|c|}
\hline Variable & Group & $\begin{array}{c}\text { Week 0 } \\
\text { (T1) }\end{array}$ & $\begin{array}{c}\text { Week } 4 \\
\text { (T2) }\end{array}$ & $\begin{array}{c}\text { Week } 8 \\
\text { (T3) }\end{array}$ & $\begin{array}{c}\text { Week 12 } \\
\text { (T4) }\end{array}$ & \multicolumn{2}{|c|}{ Significance } \\
\hline $\begin{array}{l}\text { Body } \\
\text { Weight } \\
(\mathrm{kg})\end{array}$ & $\begin{array}{l}\mathrm{CF} \\
\mathrm{P}\end{array}$ & $\begin{array}{l}87.2 \pm 12.4 \\
86.1 \pm 12.5\end{array}$ & $\begin{array}{l}86.8 \pm 12.1 \\
86.1 \pm 15.3\end{array}$ & $\begin{array}{l}86.5 \pm 12.8 \\
87.1 \pm 14.3\end{array}$ & $\begin{array}{l}86.6 \pm 13.0 \\
87.4 \pm 13.4\end{array}$ & $\begin{array}{l}\text { Group } \\
\text { Time } \\
\text { Group x Time }\end{array}$ & $\begin{array}{l}0.987 \\
0.492 \\
0.121\end{array}$ \\
\hline $\begin{array}{l}\text { Bone } \\
\text { Mineral } \\
\text { Area } \\
\left(\mathrm{cm}^{2}\right)\end{array}$ & $\begin{array}{l}\mathrm{CF} \\
\mathrm{P}\end{array}$ & $\begin{array}{l}1772 \pm 94 \\
1846 \pm 116\end{array}$ & $\begin{array}{l}1772 \pm 121 \\
1861 \pm 110\end{array}$ & $\begin{array}{l}1755 \pm 127 \\
1855 \pm 106\end{array}$ & $\begin{array}{l}1781 \pm 130 \\
1844 \pm 120\end{array}$ & $\begin{array}{l}\text { Group } \\
\text { Time } \\
\text { Group x Time }\end{array}$ & $\begin{array}{l}0.148 \\
0.929 \\
0.756\end{array}$ \\
\hline $\begin{array}{l}\text { Bone } \\
\text { Mineral } \\
\text { Content } \\
\text { (g) }\end{array}$ & $\begin{array}{l}\mathrm{CF} \\
\mathrm{P}\end{array}$ & $\begin{array}{l}1781 \pm 202 \\
1872 \pm 237\end{array}$ & $\begin{array}{l}1788 \pm 206 \\
1874 \pm 223\end{array}$ & $\begin{array}{l}1759 \pm 226 \\
1874 \pm 232\end{array}$ & $\begin{array}{l}1803 \pm 223 \\
1870 \pm 242\end{array}$ & $\begin{array}{l}\text { Group } \\
\text { Time } \\
\text { Group x Time }\end{array}$ & $\begin{array}{l}0.412 \\
0.663 \\
0.601\end{array}$ \\
\hline $\begin{array}{l}\text { Bone } \\
\text { Mineral } \\
\text { Density } \\
\left(\mathrm{g} / \mathrm{cm}^{2}\right)\end{array}$ & $\begin{array}{l}\mathrm{CF} \\
\mathrm{P}\end{array}$ & $\begin{array}{l}1.0 \pm 0.08 \\
1.0 \pm 0.08\end{array}$ & $\begin{array}{l}1.0 \pm 0.07 \\
1.0 \pm 0.07\end{array}$ & $\begin{array}{l}0.9 \pm 0.07 \\
1.0 \pm 0.08\end{array}$ & $\begin{array}{l}1.0 \pm 0.07 \\
1.0 \pm 0.08\end{array}$ & $\begin{array}{l}\text { Group } \\
\text { Time } \\
\text { Group x Time }\end{array}$ & $\begin{array}{l}0.923 \\
0.367 \\
0.578\end{array}$ \\
\hline $\begin{array}{l}\text { Fat Mass } \\
(\mathrm{kg})\end{array}$ & $\begin{array}{l}\mathrm{CF} \\
\mathrm{P}\end{array}$ & $\begin{array}{l}33.82 \pm 8.00 \\
31.27 \pm 7.22\end{array}$ & $\begin{array}{l}33.77 \pm 8.38 \\
31.69 \pm 7.94\end{array}$ & $\begin{array}{l}33.66 \pm 8.17 \\
32.20 \pm 8.70\end{array}$ & $\begin{array}{l}33.61 \pm 8.37 \\
32.36 \pm 8.22\end{array}$ & $\begin{array}{l}\text { Group } \\
\text { Time } \\
\text { Group x Time }\end{array}$ & $\begin{array}{l}0.638 \\
0.336 \\
0.161\end{array}$ \\
\hline $\begin{array}{l}\text { Lean Mass } \\
(\mathrm{kg})\end{array}$ & $\begin{array}{l}\mathrm{CF} \\
\mathrm{P}\end{array}$ & $\begin{array}{l}44.65 \pm 5.75 \\
46.40 \pm 6.18\end{array}$ & $\begin{array}{l}44.40 \pm 4.88 \\
46.47 \pm 6.45\end{array}$ & $\begin{array}{l}44.69 \pm 5.44 \\
47.01 \pm 5.97\end{array}$ & $\begin{array}{l}44.59 \pm 5.53 \\
47.05 \pm 5.62\end{array}$ & $\begin{array}{l}\text { Group } \\
\text { Time } \\
\text { Group x Time }\end{array}$ & $\begin{array}{l}0.446 \\
0.168 \\
0.212\end{array}$ \\
\hline $\begin{array}{l}\text { Lean + } \\
\text { BMC } \\
(\mathrm{kg})\end{array}$ & $\begin{array}{l}\mathrm{CF} \\
\mathrm{P}\end{array}$ & $\begin{array}{l}46.43 \pm 5.88 \\
48.27 \pm 6.36\end{array}$ & $\begin{array}{l}46.18 \pm 5.01 \\
48.35 \pm 6.63\end{array}$ & $\begin{array}{l}46.45 \pm 5.57 \\
48.88 \pm 6.16\end{array}$ & $\begin{array}{l}46.40 \pm 5.66 \\
48.92 \pm 5.81\end{array}$ & $\begin{array}{l}\text { Group } \\
\text { Time } \\
\text { Group x Time }\end{array}$ & $\begin{array}{l}0.440 \\
0.154 \\
0.211\end{array}$ \\
\hline $\begin{array}{l}\text { Total Mass } \\
(\mathrm{kg})\end{array}$ & $\begin{array}{l}\mathrm{CF} \\
\mathrm{P}\end{array}$ & $\begin{array}{l}80.25 \pm 12.42 \\
79.54 \pm 12.70\end{array}$ & $\begin{array}{l}79.96 \pm 11.93 \\
80.04 \pm 13.60\end{array}$ & $\begin{array}{l}80.11 \pm 12.59 \\
81.08 \pm 13.81\end{array}$ & $\begin{array}{l}80.017 \pm 12.7 \\
81.28 \pm 13.01\end{array}$ & $\begin{array}{l}\text { Group } \\
\text { Time } \\
\text { Group x Time }\end{array}$ & $\begin{array}{l}0.948 \\
0.139 \\
0.080\end{array}$ \\
\hline $\begin{array}{l}\text { Body Fat } \\
(\%)\end{array}$ & $\begin{array}{l}\mathrm{CF} \\
\mathrm{P}\end{array}$ & $\begin{array}{l}41.8 \pm 4.7 \\
39.0 \pm 3.7\end{array}$ & $\begin{array}{l}41.8 \pm 5.0 \\
39.5 \pm 4.0\end{array}$ & $\begin{array}{l}41.6 \pm 4.4 \\
39.2 \pm 4.6\end{array}$ & $\begin{array}{l}41.6 \pm 4.8 \\
39.3 \pm 4.3\end{array}$ & $\begin{array}{l}\text { Group } \\
\text { Time } \\
\text { Group x Time }\end{array}$ & $\begin{array}{l}0.243 \\
0.923 \\
0.395\end{array}$ \\
\hline $\begin{array}{l}\text { Body } \\
\text { Water } \\
(\%)\end{array}$ & $\begin{array}{l}\mathrm{CF} \\
\mathrm{P}\end{array}$ & $\begin{array}{l}43.9 \pm 4.0 \\
46.1 \pm 3.2\end{array}$ & $\begin{array}{l}44.1 \pm 3.6 \\
46.1 \pm 3.6\end{array}$ & $\begin{array}{l}45.5 \pm 3.8 \\
46.1 \pm 3.2\end{array}$ & $\begin{array}{l}45.3 \pm 4.3 \\
47.3 \pm 5.2\end{array}$ & $\begin{array}{l}\text { Group } \\
\text { Time } \\
\text { Group x Time }\end{array}$ & $\begin{array}{l}0.289 \\
0.200 \\
0.802\end{array}$ \\
\hline
\end{tabular}

total bilirubin, glucose, $\mathrm{BUN} /$ creatinine ratio, and cholesterol/HDL ratio within the $\mathrm{P}$ and $\mathrm{CF}$ groups. A significant decrease was noted in all of the values except for total bilirubin, which significantly increased within both groups. The cholesterol/HDL ratio also significantly increased within the $\mathrm{P}$ group.

There was a significant time effect $(p<0.05)$ in hemoglobin levels, glucose, albumin levels, red cell dimension width, and creatine kinase from Week 0 to Week 12. No significant group interactions $(p>0.05)$ were observed in the blood lipid profile, hemodynamic, hormone, insulin variables, or weekly reports of side effects. All values remained within normal clinical parameters.

Body Composition. Table 4 presents body composition data obtained during each of the four testing trials, and Figure 1 presents mean changes in body composition data from Week 0 to Week 12 . There were no significant differences $(p>0.05)$ in body weight, bone mineral area, bone mineral density, fat mass, lean mass, $\%$ body fat, or $\%$ body water observed between the two groups. In addition, there was no significant interaction $(p>0.05)$ amongst the groups in body composition. Group trends occurred in total mass $(p=0.08)$.

Psychological Profiles. A significant time effect was noted for tension/anxiety $(p=0.006)$ in the psychological analysis of mood. Group trends occurred in fatigue $(p=0.07)$ between $\mathrm{P}$ and CF from Week 0 to Week 12. No other significant differences were observed.

Appetite Profiles. Table 5 presents the appetite data obtained during each of the four testing trials. There were no significant changes observed in appetite, 

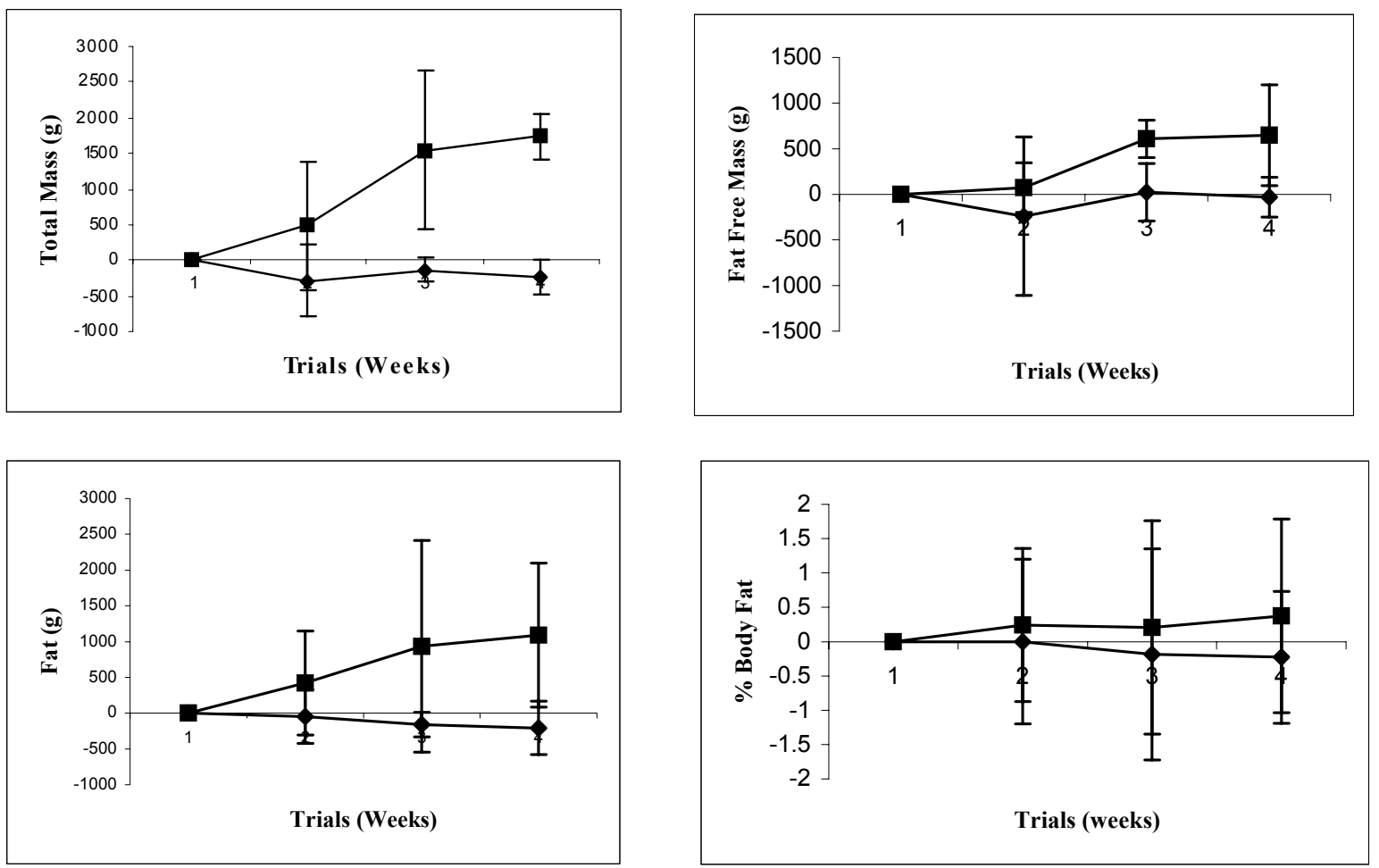

Figure 1. The upper left panel depicts changes in DEXA total scanned mass (g), the upper right panel illustrates changes in DEXA fatfree mass $(\mathrm{g})$, the lower left panel highlights differences in DEXA lean mass $(\mathrm{g})$, and the lower right panel shows changes in DEXA \% body fat from Week 0 to Week 12 for CF $(\bullet)$ and P ( $\bullet)$.

amount of energy, or overall quality of food. The analysis indicated a significant time effect $(p=$ 0.026 ) in satisfaction from food in both groups from Week 0 to Week 12. There was a significant decrease observed in the feelings of fullness variable $(p=$ 0.04) for group CF from Week 0 to Week 12.

\section{DISCUSSION}

The major findings of this study were: 1.) 12-weeks of CF supplementation ( $250 \mathrm{mg}$ of a $10 \% \mathrm{CF}$ extract taken two times per day) did not promote weight loss but may have helped mitigate weight gain in freeliving overweight females; and, 2.) CF supplementation did not adversely affect markers of health status. However, some interesting findings were observed that warrant additional research. The following discusses the results of this study in greater detail.

Dietary Intake. The subjects involved in the study recorded all food and fluid intake during Week 0 and Week 12 of the testing sessions. Significant decreases were seen in carbohydrate, fat, and energy intake for both groups over time. No significant differences were observed between $\mathrm{CF}$ and $\mathrm{P}$, which suggest that supplementation had no significant effect on diet.
The changes over time could reflect subject efforts to decrease food intake in an attempt to assist in the weight loss process. It is possible that more group differences could have been observed if a specific diet were implemented into the study. This would allow for the effects of supplementation to be more closely monitored.

Medical Safety Analysis. One purpose of this study was to determine the safety effects of forskolin supplementation on general markers of health. This was measured by monitoring changes in heart rate and blood pressure taken during each testing session, and serum and whole blood samples collected during Week 0 and Week 12 of the study. Previous research indicated that $\mathrm{CF}$ causes an increase in heart rate and a decrease in blood pressure (14-15). However, the results of this analysis showed that supplementation had no significant effect on either variable. The blood samples collected were assayed for muscle and liver enzymes, lipid profile, electrolytes, protein status, thyroid hormones, fasting insulin, and whole blood cell counts. Significant changes were observed from Week 0 to Week 12 in Group CF in white blood cell count, absolute lymphocyte count, absolute neutrophil count, calcium levels, ALT, and uric acid levels. Supplementation 
Table 5. Appetite markers for the $\mathrm{CF}$ and $\mathrm{P}$ groups.

\begin{tabular}{|c|c|c|c|c|c|c|c|}
\hline Variable & Group & $\begin{array}{c}\text { Week 0 } \\
\text { (T1) }\end{array}$ & $\begin{array}{c}\text { Week } 4 \\
\text { (T2) }\end{array}$ & $\begin{array}{c}\text { Week } 8 \\
\text { (T3) }\end{array}$ & $\begin{array}{c}\text { Week 12 } \\
\text { (T4) }\end{array}$ & \multicolumn{2}{|c|}{ Significance } \\
\hline Appetite & $\begin{array}{l}\mathrm{CF} \\
\mathrm{P}\end{array}$ & $\begin{array}{l}4.8 \pm 0.3 \\
5.1 \pm 0.8\end{array}$ & $\begin{array}{l}4.7 \pm 1.4 \\
4.1 \pm 1.6\end{array}$ & $\begin{array}{l}4.4 \pm 1.1 \\
4.3 \pm 1.4\end{array}$ & $\begin{array}{l}4.5 \pm 0.7 \\
5.1 \pm 1.1\end{array}$ & $\begin{array}{l}\text { Group } \\
\text { Time } \\
\text { Group x Time }\end{array}$ & $\begin{array}{l}0.829 \\
0.673 \\
0.581\end{array}$ \\
\hline Hunger & $\begin{array}{l}\mathrm{CF} \\
\mathrm{P}\end{array}$ & $\begin{array}{l}4.0 \pm 1.2 \\
4.9 \pm 1.1\end{array}$ & $\begin{array}{l}3.8 \pm 1.0 \\
4.0 \pm 1.5\end{array}$ & $\begin{array}{l}4.0 \pm 1.8 \\
4.1 \pm 1.1\end{array}$ & $\begin{array}{l}3.1 \pm 1.5 \\
5.2 \pm 1.0\end{array}$ & $\begin{array}{l}\text { Group } \\
\text { Time } \\
\text { Group x Time }\end{array}$ & $\begin{array}{l}0.054 \\
0.645 \\
0.165\end{array}$ \\
\hline $\begin{array}{l}\text { Satisfaction } \\
\text { from Food }\end{array}$ & $\begin{array}{l}\mathrm{CF} \\
\mathrm{P}\end{array}$ & $\begin{array}{l}5.8 \pm 1.7 \\
6.1 \pm 1.4\end{array}$ & $\begin{array}{l}5.0 \pm 0.5 \\
5.9 \pm 1.3\end{array}$ & $\begin{array}{l}4.4 \pm 1.2 \\
5.5 \pm 1.9\end{array}$ & $\begin{array}{l}4.7 \pm 1.9 \\
5.8 \pm 1.1\end{array}$ & $\begin{array}{l}\text { Group } \\
\text { Time } \\
\text { Group x Time }\end{array}$ & $\begin{array}{l}0.138 \\
0.026 \\
0.242\end{array}$ \\
\hline $\begin{array}{l}\text { Feeling of } \\
\text { Fullness }\end{array}$ & $\begin{array}{l}\mathrm{CF} \\
\mathrm{P}\end{array}$ & $\begin{array}{l}5.8 \pm 1.3 \\
6.3 \pm 1.3\end{array}$ & $\begin{array}{l}4.7 \pm 0.9 \\
5.9 \pm 1.7\end{array}$ & $\begin{array}{l}5.0 \pm 2.1 \\
6.1 \pm 1.8\end{array}$ & $\begin{array}{l}4.1 \pm 1.3 \\
6.4 \pm 1.5\end{array}$ & $\begin{array}{l}\text { Group } \\
\text { Time } \\
\text { Group x Time }\end{array}$ & $\begin{array}{l}0.040 \\
0.086 \\
0.042\end{array}$ \\
\hline $\begin{array}{l}\text { Amount of } \\
\text { Energy }\end{array}$ & $\begin{array}{l}\mathrm{CF} \\
\mathrm{P}\end{array}$ & $\begin{array}{l}5.9 \pm 2.0 \\
5.8 \pm 1.2\end{array}$ & $\begin{array}{l}5.2 \pm 1.3 \\
6.0 \pm 1.2\end{array}$ & $\begin{array}{l}5.5 \pm 2.5 \\
5.9 \pm 1.4\end{array}$ & $\begin{array}{l}5.5 \pm 1.9 \\
5.8 \pm 1.7\end{array}$ & $\begin{array}{l}\text { Group } \\
\text { Time } \\
\text { Group x Time }\end{array}$ & $\begin{array}{l}0.611 \\
0.781 \\
0.785\end{array}$ \\
\hline $\begin{array}{l}\text { Overall } \\
\text { Quality of } \\
\text { Food }\end{array}$ & $\begin{array}{l}\mathrm{CF} \\
\mathrm{P}\end{array}$ & $\begin{array}{l}4.8 \pm 1.5 \\
5.2 \pm 1.4\end{array}$ & $\begin{array}{l}5.1 \pm 1.8 \\
5.7 \pm 1.0\end{array}$ & $\begin{array}{l}4.5 \pm 1.1 \\
5.6 \pm 1.3\end{array}$ & $\begin{array}{l}4.0 \pm 1.7 \\
5.6 \pm 1.4\end{array}$ & $\begin{array}{l}\text { Group } \\
\text { Time } \\
\text { Group x Time }\end{array}$ & $\begin{array}{l}0.102 \\
0.472 \\
0.134\end{array}$ \\
\hline
\end{tabular}

resulted in an increase in calcium, white blood cell, absolute lymphocyte, and absolute neutrophil counts. Decreases were observed in ALT and uric acid levels. These variables contribute to muscle, immune, liver, and protein functions, respectively, in the body. Even though these changes occurred, the values remained within normal ranges and were relatively small. Additionally, no significant adverse events were reported during the study that could be attributed to the supplementation protocol. Therefore, these findings suggest that $\mathrm{CF}$ supplementation does not appear to be associated with any significant clinical side effects.

Body Composition. This study was also conducted with the purpose of investigating the effectiveness of $\mathrm{CF}$ on the management of body composition. Body composition was analyzed by examining measures of body weight, body water, and bone density. Previous research suggests that forskolin is associated with increased lipolysis, which would increase fat and weight loss in the body (13). The findings from the previous pilot study suggested that supplementation significantly decreases body weight and fat, and significantly increases lean body mass in free living subjects (5). The results from this study did not replicate those findings. Additionally, results contrast recent findings by Godard and colleagues (6) who reported that 12 -weeks of CF $(250 \mathrm{mg}$ of a $10 \%$ CF preparation taken two times per day) promoted favorable changes in body composition in overweight men. In the present study, no significant changes were observed in body weight, fat content, or lean body mass. However, statistical trends were found in total scanned mass, which could suggest some alleviation in weight gain among overweight subjects. Overall, CF had no effect on the fat free or fat mass of the female subjects used in the study. It is possible that more group differences could be seen if controls for exercise and/or diet were incorporated and/or the study was conducted in a larger population.

Psychological Analysis. A psychometric inventory was used to analyze mood changes during each of the four testing sessions of the study. This analysis measured potential changes in six different mood states: tension-anxiety, depression-dejection, angerhostility, vigor-activity, fatigue-inertia, and confusion-bewilderment. The results of this psychological inventory revealed that regular ingestion of the supplement produced no significant alteration in mood, except for the decrease in tensionanxiety over time. This decrease could be associated with the possible effects of supplementation on body weight, which is an issue that often leads to tensionanxiety. Trends were observed in fatigue levels. Subjects taking the supplement appeared to experience less fatigue over the course of the study. Because there were no controls for physical activity in this study, only speculation can explain the changes that occurred. It is possible that those in CF engaged in less activity with the idea that supplementation would increase weight loss. 
Appetite Analysis. An appetite inventory was used to assess variables of appetite during each of the four testing sessions of the study. This inventory measured changes in hunger, appetite, satisfaction from food, fullness, and overall quality of food. No significant differences were observed in appetite, hunger, energy, or overall quality of food. This shows that the eating desires for both groups were maintained. However, the significant time effect in satisfaction from food found in $\mathrm{CF}$ and $\mathrm{P}$ suggests that both groups received less enjoyment from the food consumed. There was also a significant decrease noted in feelings of fullness in CF from Week 0 to Week 12. Even though appetite and hunger were not significantly affected by supplementation, it is possible that because $\mathrm{CF}$ received less satisfaction from food that less food was eaten.

Summary. In conclusion, this study was performed in an attempt to further investigate the effectiveness of $\mathrm{CF}$ on body composition and general markers of health. The findings suggest that 12-weeks of $\mathrm{CF}$ supplementation in free-living subjects have limited effects on body composition and no apparent clinically significant side effects. However, CF has the potential to positively influence the loss and management of overall body weight possibly as a means of maintaining weight after weight loss. Still, future research is needed to further explore its capabilities.

\section{ACKNOWLEDGEMENTS}

This study was conduced at University of Memphis when the primary researchers were affiliated with that institution. We would like to thank the subjects who participated in this study and the laboratory assistants in Exercise \& Sport Nutrition Laboratory at The University of Memphis who assisted in data acquisition and researchers in the Exercise \& Sport Nutrition Laboratory at Baylor University who assisted with data analysis and manuscript preparation.

This study was funded in part by a research grant from Sabinsa Corporation (Piscataway, NJ) in collaboration with ImagiNutrition (Laguna Niguel, $C A$ ). Investigators independently collected, analyzed and interpreted data from this study and have no financial interest in the outcome of results reported. Presentation of results in this study does not constitute endorsement by researchers or their institutions of the supplement investigated.

\section{REFERENCES}

1. Agarwal, K.C., R.E. Parks. Forskolin: a potential antimetastic agent. Int J Cancer. 32:801-804, 1983.

2. Caprioli, J., M. Sears. Forskolin lowers intraocular pressure in rabbits, monkeys, and man. Lancet. 958-960, April 30, 1983.

3. Burns, T.W., Langley, P.E., Terry, B.E., D.B. Bylund, L.R. Forte, Jr. Comparative effects of forskolin and isoproterenol on the cyclic AMP content of human adipocytes. Life Sci. 40: 145-54, 1987.

4. Litosch, I., Hudson, T.H., Mills, I., Li, S.Y., J.N. Fain. Forskolin as an activator of cyclic AMP accumulation and lipolysis in rat adipocytes. Mol Pharmacol. 22:109-15, 1982.

5. Diterpene forskolin (Coleus forskohlii, Benth.): A Possible New Compound for Reduction of Body Weight by Increasing Lean Body Mass Database [http://www.forlslean.com/clinical_studies.html]

6. Godard, M.P., Johnson, B.A., S.R. Richmond. Body Composition and Hormonal Adaptations Associated with Forskolin Consumption in Overweight and Obese Men. Obesity Research. 13:1335-1343, 2005.

7. Srivasta, S.K., Khatoon, CS, Mehrotra, S, R. Mehrotra. Pharmacognistic evaluation of coleus forskohlii. Pharmaceutical Biology. 40(2):129-134, 2002.

8. Exercise and Sport Nutrition: A Balanced Perspective for Exercise Physiologists Database. [http://www3.baylor.edu/HHPR/faculty/Kreider/Courses/PEPNutrition.pdf]

9. Flint, A., A. Raben, et al. Reproducibility, power and validity of visual analogue scales in assessment of appetite sensations in single test meal studies. Int J Obes Relat Metab Disord. 24(1): 38-48, 2000.

10. McNair, D.M., M. Loor, L.F. Droppleman. Edits Manual for the Profile of Mood States. San Diego Educational and Industrial Testing Service, 1992.

11. Almada, A.L., R.B. Kreider, et al. Day-To-Day Reliability in Spine, Hip, and Whole Body DEXA Scans in Men and Women. Southeast American College of Sports Medicine Conference Abstracts. 27: 36, 1999.

12. Franklin, B., M. Whaley (Eds.). ACSM's Guidelines for Exercise Testing and Prescription. Philadelphia: Lippincott Williams \& Wilkins; 2000.

13. Kreider, R.B., M. Ferreira, et al. Effects of Calcium $\beta$-hydroxy $\beta$-methylbutyrate (HMB) Supplementation During ResistanceTraining on Markers of Catabolism, Body Composition and Strength. Int J Sports Med. 20: 1-7, 1999.

14. Rupp, R.H., N.J. de Souza, A.N. Dohadwalla. Proceedings of the international symposium on Forskolin: Its chemical, biological and medical potential. Organized by Hoechst Centre for Basic Research, Bombay on January 28-29, 1985. Hoechst India Limited.

15. Seamon, K.B., W. Padgett, J.W. Daly. Forskolin: unique diterpene activator of adenylate cyclase in membranes and in intact cells Proc Natl Acad Sci U S A. 78:3363-7, 1981.

16. Kasonia K. Preliminary screening of plant extracts used in respiratory pathology in Kivu/Zaire on isolated guinea pigs rings trachea. Belgian Journal of Botany. 128:165-175, 1995.

17. Bauer, K. et al. Pharmacodynamic effects of inhaled dry powder formulations of fenoterol and colforsin in asthma. Clin. Pharmacol. Ther. 53:76-83, 